\title{
Un nouveau locus impliqué dans la détermination sexuelle ou comment SRY n'explique pas tout...
}

Il était bien normal que la découverte d'un locus probablement impliqué dans la détermination sexuelle féminine revînt à une femme... Que l'équipe soit presque exclusivement italienne ajoute encore au charme...

Voici donc l'équilibre rétabli : le gène $S R Y$ n'est plus le facteur omnipotent de la détermination masculine et, enfin, nous ne sommes plus "femme" à défaut d'être "homme»!

En réalité, l'implication dans la détermination sexuelle humaine d'un gène lié à l'X avait été suspectée dès 1978 après l'observation d'une inversion sexuelle familiale transmise par le chromosome X. L'équipe italienne a regroupé 31 de ces inversions sexuelles, c'est-à-dire de discordances entre un génotype masculin $(46, \mathrm{XY})$ et un phénotype féminin [1]. Parmi celles-ci, on compte quatre duplications d'une partie du chromosome $\mathrm{X}$ chez des patients qui présentent un tableau clinique complexe, associant des malformations mineures à une dysgénésie gonadique, c'est-à-dire un développement anormal des gonades détecté à l'examen histologique.

Par Southern blot, cette équipe a tout d'abord montré que les points de cassure de ces duplications étaient différents, détruisant l'hypothèse de l'interruption d'un gène à l'origine des inversions sexuelles. En revanche, cette observation est un fort argument pour penser que le changement de sexe est dû à un déséquilibre de dosage génique : deux doses du locus, désormais appelé $D S S$ (pour dosage sensitive sex reversal), contre une seule dose du gène $S R Y$ suffisent à empêcher un développement testiculaire normal et à orienter le développement gonadique vers la formation d'un ovaire. quatre cas de duplication partielle ont été comparés à quatre autres duplications du bras court du chromosome $\mathrm{X}$ non associées à une inversion sexuelle: ainsi, la région minimale dupliquée et entrainant un changement phénotypique de sexe a-t-elle pu être établie à 20 mégabases. Il a ensuite été supposé que dans les vingt-sept autres cas d'inversion sans anomalie caryotypique a priori, il existait des réarrangements submicroscopiques dans la région préétablie. L'équipe a donc recherché, par dosage génique, la présence d'une duplication. Par chance, l'ADN d'un des patients a révélé une duplication d'environ une mégabase, proche du locus de la dystrophine en Xp21. La région minimale de chevauchement des duplications donnant lieu à un changement de sexe a alors pu être réestimée à $160 \mathrm{~kb}$. Cette région est très proche de, voire confondue avec, celle du locus de l'hypoplasie congénitale des surrénales $(C A H)$. L'étau se resserre et l'isolement dans une si petite région d'ADN du gène DSS semble imminent ; il pourrait même s'agir du gène $C A H$ lui-même qui, en cas de délétion, empêcherait la différenciation des glandes surrénales et, en cas de duplication, empêcherait la différenciation testiculaire.

Quoi qu'il en soit, le mécanisme d'action du gène DSS n'est pas encore connu; on peut tout au plus suggérer que ce gène contrebalance l'effet du gène $S R Y$ puisqu'un double dosage de DSS empêche la formation d'un testicule chez un sujet de caryotype XY alors qu'il n'induit pas d'anomalie ovarienne majeure chez un sujet de caryotype XX (les femmes présentant cette duplication sont en effet fertiles). Le gène $D S S$ est peut-être impli- qué dans la détermination gonadique ovarienne mais probablement pas dans la détermination testiculaire puisque certains patients XY atteints d'hypoplasie congénitale des surrénales et présentant une délétion du locus DSS ont un développement externe génital mâle.

De même, le profil d'inactivation de ce gène n'a pas encore été étudié. Bien que situé en dehors de la région pseudoautosomique du chromosome $\mathrm{X}$, région qui n'est pas soumise à l'inactivation, il pourrait néanmoins échapper à ce processus. Dans le cas contraire, son existence ne suffirait pas à expliquer la dysgénésie gonadique du syndrome de Klinef elter qui possède, comme l'homme normal, un seul $\mathrm{X}$ actif et un gène $S R Y$, ni l'atrophie ovarienne du syndrome de Turner, où le gène DSS est $a$ priori présent en une seule copie, comme chez la femme normale. Peut-on, pour autant, impliquer d'autres gènes sur le chromosome $\mathrm{X}$ dont le dosage génique serait soigneusement contrôlé pour donner lieu à un développement gonadique normal?

Finalement, quel que soit le profil d'inactivation du gène $D S S$, que les sexistes se rassurent : dans le schéma qui semble se dessiner, le gène $S R Y$ reste capable d'inhiber la fonction du gène DSS lorsque celui-ci n'est présent qu'en une seule copie!

H.G.

1. Bardoni B, Zanaria E, Guioli S, Floridia G, Worley KC, Tonini G, Ferrante E, Chiumello G, McCabe FRB, Fraccaro M, Zuffardi O, Camerino G. A dosage sensitive locus at chromosome Xp2l is involved in male to female sex reversal. Nature Genet $1994 ; 7: 497-501$. 